\title{
BMJ Open Rationale and design of a multicentre, prospective, randomised, controlled clinical trial to evaluate the efficacy of the adipose graft transposition procedure in patients with a myocardial scar: the AGTP II trial
}

Paloma Gastelurrutia, ${ }^{1,2}$ Carolina Gálvez-Montón, ${ }^{1,2}$ Maria Luisa Cámara, ${ }^{3}$ Juan Bustamante-Munguira, ${ }^{2,3,4}$ Pablo García-Pavia, ${ }^{2,5}$ Pablo Avanzas, ${ }^{6}$ J Alberto San Román,, ${ }^{2,7}$ Domingo Pascual-Figal, ${ }^{2,8}$ Eduardo de Teresa, ${ }^{2,9}$ Maria G Crespo-Leiro, ${ }^{2,10}$ Nicolás Manito, ${ }^{11}$ Julio Núñez, ${ }^{2,12}$

Francisco Fernández-Avilés, ${ }^{2,13}$ Ángel Caballero, ${ }^{3}$ Albert Teis, ${ }^{3}$ Josep Lupón, ${ }^{2,3,14}$ Ramón Brugada, ${ }^{2,4}$ Carlos Martín, ${ }^{5}$ Jacobo Silva, ${ }^{6}$ Ana Revilla-Orodea, ${ }^{2,7}$ Sergio J Cánovas, ${ }^{8}$ Jose M Melero, ${ }^{2,9}$ Jose J Cuenca-Castillo, ${ }^{2,10}$ Angel Gonzalez-Pinto, ${ }^{13}$ Antoni Bayes-Genis ${ }^{1,2,3,14}$

To cite: Gastelurrutia P, Gálvez-Montón C, Cámara ML, et al. Rationale and design of a multicentre, prospective, randomised, controlled clinical trial to evaluate the efficacy of the adipose graft transposition procedure in patients with a myocardial scar: the AGTP II trial. BMJ Open 2017;0:e017187. doi:10.1136/ bmjopen-2017-017187

- Prepublication history for this paper is available online. To view these files please visit the journal online (http://dx.doi org/10.1136/bmjopen-2017017187).

Received 11 April 2017

Revised 8 June 2017

Accepted 5 July 2017

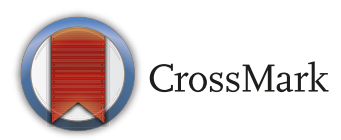

For numbered affiliations see end of article.

Correspondence to Dr Paloma Gastelurrutia; pgastelurrutia@gmail.com

\section{ABSTRACT}

Introduction Cardiac adipose tissue is a source of progenitor cells with regenerative capacity. Studies in rodents demonstrated that the intramyocardial delivery of cells derived from this tissue improves cardiac function after myocardial infarction (MI). We developed a new reparative approach for damaged myocardium that integrates the regenerative properties of cardiac adipose tissue with tissue engineering. In the adipose graft transposition procedure (AGTP), we dissect a vascularised flap of autologous pericardial adipose tissue and position it over the myocardial scarred area. Following encouraging results in acute and chronic MI porcine models, we performed the clinical trial (NCT01473433, AdiFLAP trial) to evaluate safety in patients with chronic MI undergoing coronary artery bypass graft. The good safety profile and trends in efficacy warranted a larger trial.

Study design The AGTP II trial (NCT02798276) is an investigator initiated, prospective, randomised, controlled, multicentre study to assess the efficacy of the AGTP in 108 patients with non-revascularisable MI. Patients will be assigned to standard clinical practice or the AGTP. The primary endpoint is change in necrotic mass ratio by gadolinium enhancement at 91 and 365 days. Secondary endpoints include improvement in regional contractibility by MRI at 91 and 365 days; changes in functional MRI parameters (left ventricular ejection fraction, left and right ventricular geometric remodelling) at 91 and 365 days; levels of $\mathrm{N}$-terminal prohormone of brain natriuretic peptide (NT-proBNP) at 7, 91 and 365 days; appearance of arrhythmias from 24 hour Holter monitoring at 24 hours, and at 91 and 365 days; all cause death or re-hospitalisation at 365 days;
Strengths and limitations of this study:

- The adipose graft transposition procedure (AGTP) is the first tissue engineering technique to use local adipose tissue without laboratory manipulation.

- It is technically simple, requiring no learning curve for cardiac surgeons who will simply dissect structures that are ordinarily modified in coronary artery bypass graft surgery.

- There is no requirement for additional or expensive materials, nor do any ethical or social constraints exist as the autologous tissue is sourced from a site in close proximity to the injury.

- It minimises the complications that can arise from the introduction of exogenous stem cells or stem cell derived cardiomyocytes, as well as those associated with gene therapy.

- Access to local adipose tissue is also a critical aspect of the AGTP because its feasibility may be limited in patients with very little pericardial fat.

and cardiovascular death or re-hospitalisation at 365 days.

Ethics and dissemination The institutional review board approved the trial which will comply with the Declaration of Helsinki. All patients will provide informed consent. It may offer a novel, effective and technically simple technique for patients with no other therapeutic options. The results will be submitted to indexed medical journals and national and international meetings.

Trial registration number ClinicalTrials.gov: NCT02798276, pre-results. 


\section{RATIONALE}

\section{Cardiac adipose tissue for heart regeneration}

The regenerative capacity of the heart is limited. After a myocardial infarction (MI), there is massive loss of cardiac muscle that overloads the surviving myocardium and ultimately leads to heart failure. ${ }^{1}$ Our group, ICREC Research Program, has focused on cardiac adipose tissue as a source of progenitor cells with regenerative capacity, and as a biological matrix for salvaging injured myocardium following MI. Under physiological conditions, cardiac adipose tissue displays biochemical and thermogenic cardioprotective properties. Further, during pathological scenarios, cardiac adipose tissue can locally affect the heart via the vasocrine or paracrine secretion of proinflammatory cytokines. $^{23}$

We identified and characterised a population of human adult mesenchymal-like cells derived from cardiac adipose tissue (cardiac adipose tissue mesenchymal stem cells (cATMSCs)). Despite residing in an adipocytic environment, cATMSCs have an inherent cardiac-like phenotype and may play a role in heart homeostasis as well as act as a cellular reservoir for myocardial tissue renewal. In vivo studies in murine models showed that the intramyocardial delivery of cATMSCs improves cardiac function, reduces infarct size and avoids infarct related reduction of wall thickness following MI. ${ }^{4}$ Moreover, following the application of cATMSCs, cellular niches within the host myocardium secrete pro-angiogenic factors and differentiate into an endothelial lineage that can support local angiogenesis. Collectively, these preliminary results encouraged us to expand the therapeutic uses of cATMSCs.

\section{A new approach: preclinical trials}

Our next aim was avoiding any damage that could be caused by intramyocardial injection or the use of cellular material from allogeneic sources. Consequently, we developed a new approach that integrates cATMSC therapy with tissue engineering to repair damaged myocardium (figure 1). This innovative procedure involves the dissection of a vascularised flap of autologous pericardial adipose tissue to cover the infarcted myocardium.

Initially, we tested this strategy, named the adipose graft transposition procedure (AGTP), in a porcine acute MI model. After a lateral thoracotomy to expose the pericardium, adipose tissue from the fibrous pericardium was carefully dissected, while maintaining its vascularisation, to obtain a natural scaffold containing native

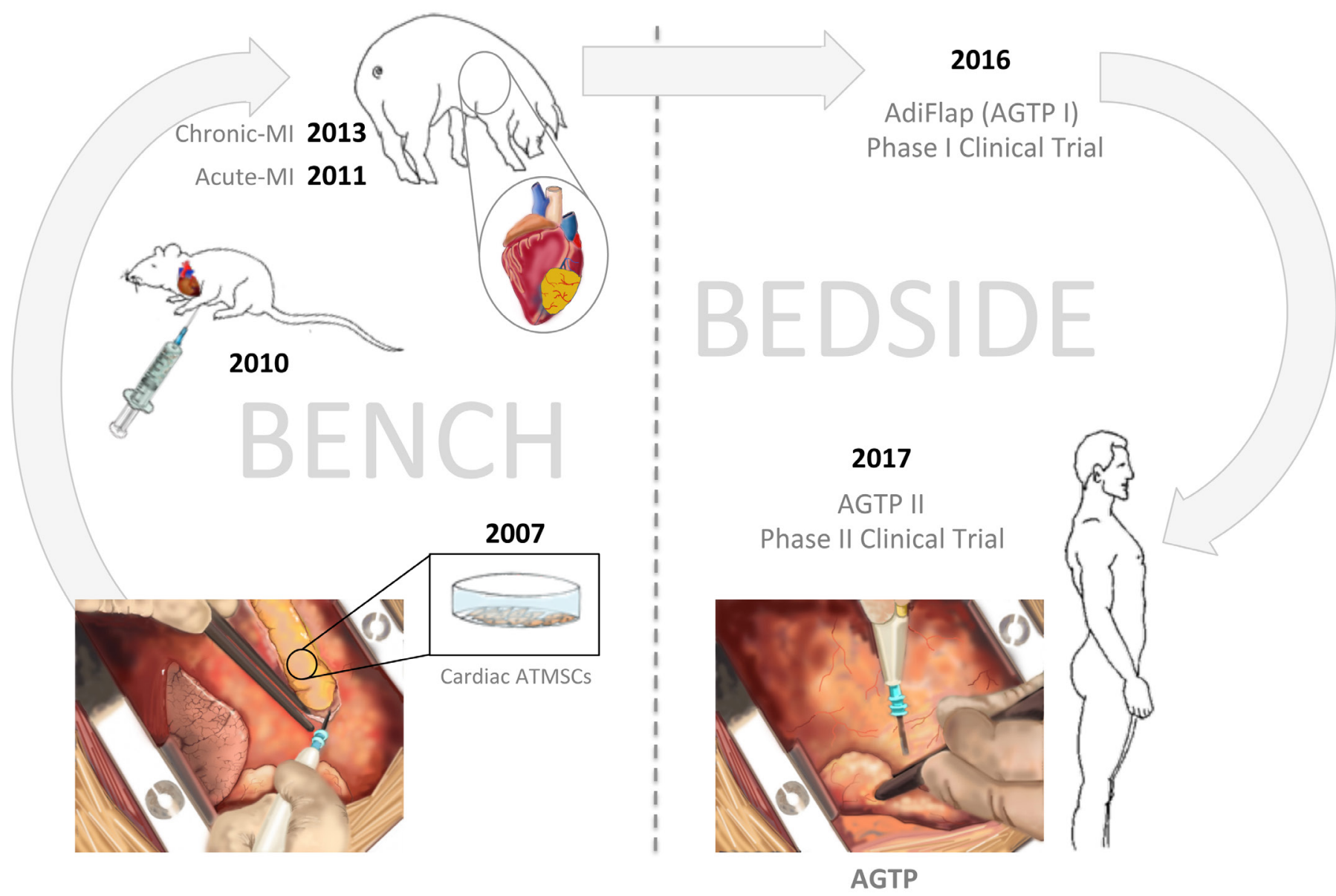

Figure 1 Schematic illustration of the use of adipose tissue mesenchymal stem cells and the development of the adipose graft transposition procedure in preclinical and clinical studies. The figure was designed and hand drawn by CG-M. AGTP, adipose graft transposition procedure; ATMSCs, adipose tissue mesenchymal stem cells. MI, myocardial infarction. 
cATMSCs. The flap was reserved and a pericardiotomy was performed to access the heart. After MI induction by permanent coronary artery ligation, the adipose pericardial graft was adhered to healthy myocardium fully covering the ischaemic area. At the 30 day follow-up, we observed that AGTP treated animals manifested a fivefold improvement in the left ventricular ejection fraction (LVEF) compared with control animals, and a smaller infarct size and myocardial remodelling. ${ }^{5}$ The AGTP was performed $30 \mathrm{~min}$ after MI induction (an acute scenario not used in the clinical setting), and thus we also re-tested the AGTP in a chronic MI swine model, positioning the flap 2 weeks after coronary coil deployment. Once again, the AGTP proved valuable in reducing the left ventricular infarct area by as much as $34 \%$ versus control animals, and in promoting neovascularisation in the infarcted myocardium. ${ }^{6}$ Microscopic evaluation revealed that both models promoted vascular bridging between the ischaemic myocardium and the adipose graft. In terms of safety, the AGTP did not trigger arrhythmic events or the sudden death of any of the included animals. Therefore, we proceeded with further bench to bedside studies.

\section{Safety clinical trial}

The AGTP I clinical trial (NCT01473433, AdifLAP trial) was designed to evaluate the safety of the AGTP in patients with chronic MI undergoing a coronary artery bypass graft $(\mathrm{CABG})$ for other myocardial regions. ${ }^{7} \mathrm{~Pa}-$ tients were enrolled if they were CABG candidates and had an established, chronic, MI lesion that was not in the area supplied by the CABG target vessel(s). Patients were assessed with late gadolinium enhancement (LGE) MRI to identify a chronic non-revascularisable area to guide the AGTP. The main safety endpoints were arrhythmia, hospital admission and death during the 12 month follow-up period. Secondary endpoints included change in cardiac geometry and function, as determined by cardiac MRI, biomarkers of cardiac injury or strain (eg, troponin $\mathrm{T}$ and $\mathrm{N}$-terminal prohormone of brain natriuretic peptide (NT-proBNP)), NYHA class and Framingham derived clinical score. ${ }^{7}$

Ten male patients were recruited from January 2012 to November 2013 in a single centre. All were candidates for myocardial revascularisation with an established chronic transmural myocardial scar in a distally non-revascularisable area. They did not differ in disease severity or surgical risk variables, with a necrotic non-revascularisable mass of $34 \pm 13 \mathrm{~g}$ versus $25 \pm 4 \mathrm{~g}$ and an LVEF value of $41 \% \pm 18 \%$ versus $42 \% \pm 15 \%$ in the AGTP treated versus the control arm, respectively. Patients were randomly allocated to either the control or AGTP treated arm; for both groups, the area that could be revascularised was treated with CABG. The non-revascularisable scar was left untouched in the control arm and was covered by an autologous pericardial adipose graft in the AGTP arm. The AGTP location was inferior in four cases and anterior in one. No differences in safety were observed in terms of clinical or arrhythmic events, as assessed by clinical visits, ECG and 24 hour Holter monitoring during the 12 month study duration. On follow-up, the AGTP treated arm showed a borderline smaller left ventricular end systolic volume (LVESV; $\mathrm{p}=0.09$ ) and necrosis ratio, assessed with LGE MRI $(p=0.06)$. Interestingly, the AGTP patient with the largest necrotic area and most dilated chambers experienced the most remarkable reduction in necrotic mass size $(-10.8 \%)$ and ventricular volumes (left ventricular end-diastolic volume (LVEDV): $-55.2 \mathrm{~mL}$ and LVESV: $-37.8 \mathrm{~mL}$ ) at the 1 year follow-up. Furthermore, this patient's $Q$ waves vanished from leads I, II and III in the follow-up ECGs. Based on these results and the good safety profile of the AGTP, we are undertaking a larger trial to assess the efficacy of the AGTP.

\section{DESIGN AND METHODS \\ Study aim}

To compare the efficacy of the AGTP with standard clinical practice in reducing a non-revascularisable MI at 3 months and/or 1 year in patients undergoing CABG of other distal myocardial areas.

\section{Primary hypothesis}

The AGTP will be able to reduce the necrotic mass ratio of a non-revascularisable MI, defined as a reduction of $10 \%$ in the estimated infarct size, as determined by LGE MRI at 91 and/or at 365 days in patients undergoing CABG of other myocardial areas.

\section{Secondary hypothesis}

- Compared with those receiving the standard clinical practice, patients undergoing the AGTP will show:

- Better regional contractibility

- Increased LVEF

- Less remodelling (assessed by MRI)

- Lower levels of biomarkers (NT-proBNP and high sensitive troponin $\mathrm{T}$ )

- The AGTP will be safe with no increase in arrhythmias (24hour Holter monitoring) or outcomes (all cause death or readmission, cardiovascular death or readmission).

\section{Trial population}

Men or women aged $\geq 18$ years, with a $Q$ wave in the ECG and a history of a transmural MI ( $\geq 50 \%$ late gadolinium enhancement) that is anatomically non-amenable to surgical revascularisation, who are candidates for CABG of other myocardial areas will be included and randomised after they agree to participate and provide signed informed consent. Exclusion criteria include severe valvular disease that could be repaired surgically, candidacy for surgical ventricular remodelling, contraindications for MRI, extracardiac disease with a vital prognosis of $<1$ year, severe renal or hepatic failure, previous cardiac surgery, pregnancy or breastfeeding. 


\section{Trial design}

The AGTP II trial (NCT02798276) is an investigator initiated, prospective, randomised, controlled, multicentre (11 tertiary hospitals in Spain) study comparing two arms of patients with chronic MI undergoing CABG with a distally non-revascularisable area. The protocol (V.2.2, 4 April 2017) has been approved by the corresponding institutional review board. A total of 108 patients with a myocardial scar who are candidates for revascularisation of other myocardial areas will be included at participating sites in Spain by the cardiology and cardiac surgery teams. The selected hospitals have a strong clinical cardiology and cardiac surgery team prepared to work together, along with adequate facilities for cardiac MRI. The AGTP II trial has currently been enrolling patients since 1 May 2017.

\section{Randomisation and blinding}

After providing informed consent to the clinical professional, patients will be centrally randomised at a 1:1 ratio into two arms by allocation order. The randomisation scheme was computer generated by statisticians from the IMIM (Barcelona, Spain), and once a patient is enrolled in the study, the local surgeon or study coordinator will contact the coordinator centre to be provided with the allocation group. Treatment allocation will be blind to the patient and to the clinical team that performs the follow-up.

\section{Study procedure and follow-up}

The AGTP will be taught to other cardiac surgeons at study centres by a cardiac surgeon (MLC) ${ }^{3}$ skilled in the procedure who performed all surgeries in the AGTP I trial. Patients will be included into two arms, stratified according to the age of the scar (figure 2).

A. For treated AGTP patients $(n=54)$ : the surgeon will perform a sternotomy for the CABG of revascularisable vessel(s). The pericardial tissue will be gently dissected while maintaining its functional vascularisation. The pericardium will be excised, and the adipose flap fixed over the nonrevascularisable scar using surgical glue. Patients randomised to this AGTP group without sufficient fat tissue within the flap will be excluded from the study, and analysed in a subgroup.

B. For control patients $(n=54)$ : the surgeon will perform a sternotomy for the CABG of the revascularisable $\operatorname{vessel(s).~The~distally~non-revascularisable~area~will~}$ be left untouched (the current standard of care).

Both groups will be hospitalised for the usual time after a CABG. In the AGTP I trial, we found that the AGTP does not prolong inhospital stay. To ensure adequate assessment of efficacy, all patients will undergo several tests (see table 1), including MRI, ECG, 24 hour Holter monitoring and blood tests. A clinical cardiologist blind to the randomisation group will conduct the follow-up visits (table 1). Additional patient visits can also be made, if required, at the discretion of the clinical team. Cardiac MRI will be performed in a standardised manner at each participant centre with $1.5 \mathrm{~T}$ equipment and assessed by a centralised core lab (ICICORELAB, Valladolid, Spain). All images will be captured under ECG monitoring and in apnoea. Images will be obtained with three plane locators. For the study of ventricular function, we will obtain steady state free precession sequences (TrueFISP, bTFE, FIESTA) at short axis ( $8 \mathrm{~mm}$ slices from the apex to the mitral ring), four chamber and two chamber views. For the study of myocardial fibrosis/necrosis, a gadolinium based contrast agent will be intravenously administered and the necrotic myocardium will be assessed using delayed enhancement. The necrotic size and the salvaged myocardium will be expressed as a percentage of the total left ventricular mass. In addition, the necrotic area will be expressed in grams. All results will be given in absolute numbers and normalised by body surface area. In addition, LVESV and LVEDV will be assessed. Interobserver and intraobserver variability will be assessed using randomly chosen (15\%) MRI studies.

\section{Risks and benefits}

This clinical study is designed and will be implemented in accordance with the Declaration of Helsinki, with all patients providing informed consent and patients' data being anonymised. The study has been assessed and approved by the hospital ethics committee (reference number AC-16-025). Based on the preclinical and clinical AGTP phase I study, no safety issues are anticipated. Potential perioperative risks include pericardium dissection and pericardial fat manipulation, both of which are normal processes in conventional CABG. Surgical glue (Glubran2, GEM srl, Viareggio, Italy) is routinely used in surgeries, including conventional cardiac interventions in clinical practice. Moreover, a high sensitivity troponin $\mathrm{T}$ curve will be obtained during the surgical procedure to assess the patient's well being. Perioperative infarct will be assessed according to the published definition of CABG related MI. ${ }^{8}$ Finally, despite no arrhythmic events detected in the AGTP I trial, patients will be closely monitored during their hospital admission. When discharged, the first visit will be 1 week post-discharge, with follow-up extending to 1 year. Patients will be advised to contact the clinical team in case of any discomfort. Patients are covered by an insurance policy. Based on preclinical results, the expected benefits of the AGTP II trial include infarct size reduction and an improvement in cardiac function. These benefits may be detected by MRI, changes in surrogate biomarker concentration and by clinical outcomes.

\section{Criteria for study withdrawal}

Patients may voluntarily leave the study at any time without giving any reason and with no consequences for their medical care. They will also be withdrawn if any exclusion criterion is detected after informed consent is obtained, if they are lost to follow-up or fail to collaborate. However, irrespective of the reason for withdrawal, the investigators will make all efforts to perform the end of study visit and collect all available information from the patient. 


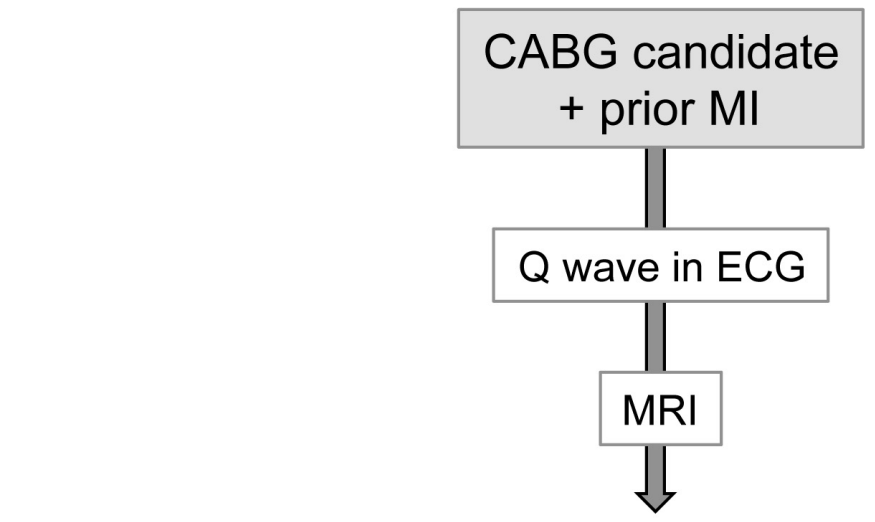

Transmural scar with LGE $\geq 50 \%$
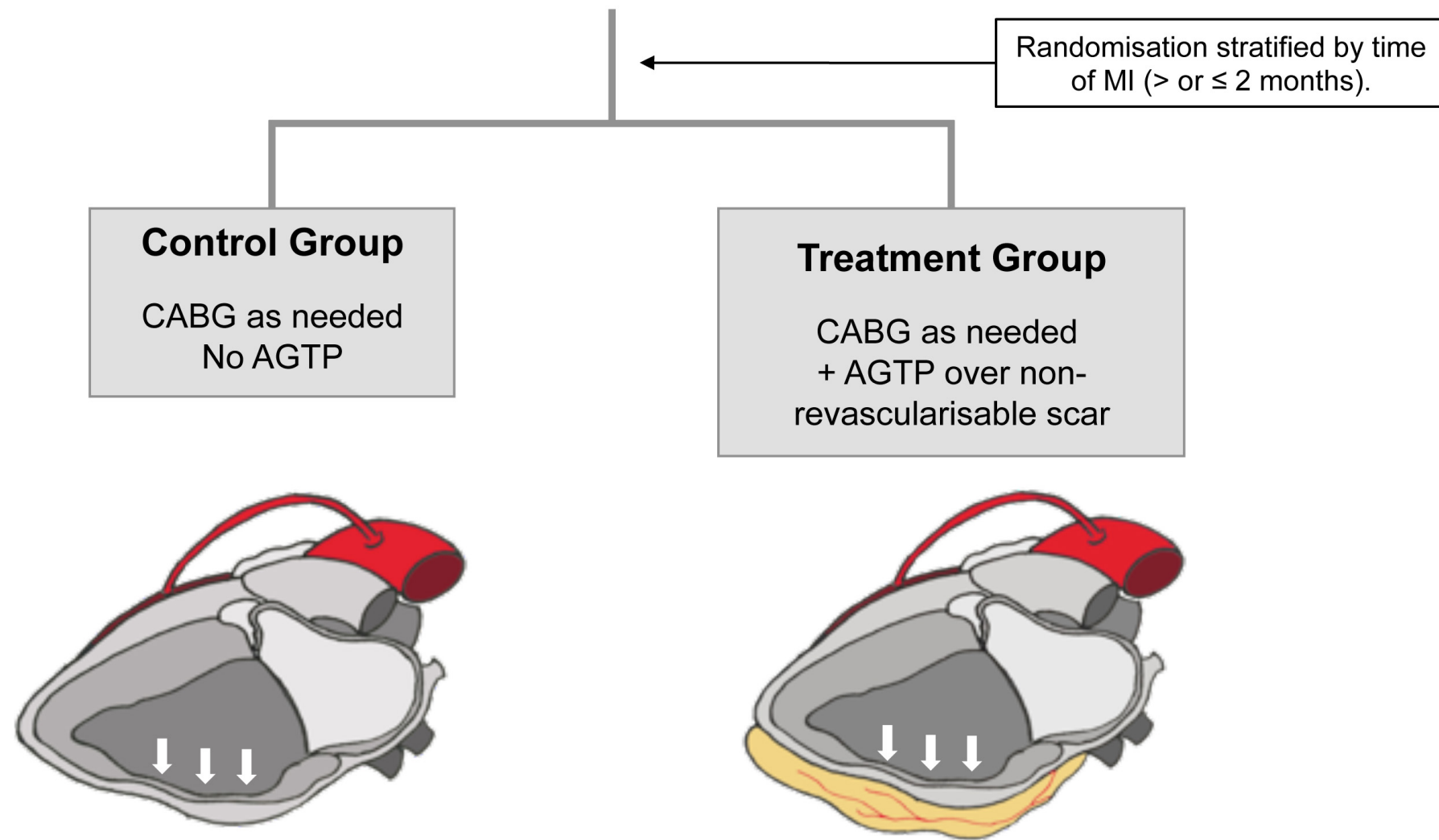

Figure 2 Flowchart of the clinical trial design of the adipose graft transposition procedure (AGTP) II trial showing the procedures performed in the two arms of the study. Arrows indicate the infarcted area covered by the AGTP in the treatment group. The figure was designed and hand drawn by CG-M. CABG, coronary artery bypass graft; LGE, late gadolinium enhancement; Ml, myocardial infarction.

\section{Trial outcomes}

The main outcome is the proportion of patients who have a smaller necrotic mass ratio of a non-revascularisable MI, defined as a reduction of $10 \%$ in the estimated infarct size determined by LGE MRI at 91 and/or 365 days compared with the baseline assessment ( $\%$ and/or grams).

Secondary outcomes:

- Improvement in regional contractibility by MRI (time frame 0-3-12 months)
Percent changes in functional parameters by MRI: LVEF, right ventricular ejection fraction (time frame 0-3-12 months)

- Changes $(\mathrm{mL})$ in ventricular volumes by MRI: LVESV, LVEDV, right ventricular end systolic volume, right ventricular end diastolic volume (time frame 0-3-12 months)

- Changes $(\mathrm{L} / \mathrm{min})$ in cardiac output by MRI (time frame 0-3-12 months)

- Changes in levels of natriuretic peptides (time frame 0-1 week and 3-12 months) 
Table 1 Study visits

\begin{tabular}{|c|c|c|c|c|c|}
\hline & $\begin{array}{l}\text { Selection and } \\
\text { randomisation }\end{array}$ & Surgery & Follow-up & & \\
\hline Visit & 1 & 2 & 3 & 4 & 5 \\
\hline Time & 0 & 0 & $\begin{array}{l}1 \text { week post- } \\
\text { discharge } \\
( \pm 7 \text { days })\end{array}$ & $\begin{array}{l}\text { 3months post- } \\
\text { discharge } \\
\text { ( } \pm 7 \text { days) }\end{array}$ & $\begin{array}{l}1 \text { year post- } \\
\text { discharge } \\
\text { ( } \pm 15 \text { days) }\end{array}$ \\
\hline Informed consent & $\mathrm{x}$ & & & & \\
\hline Inclusion/exclusion criteria & $x$ & & & & \\
\hline Vital signs & $x$ & $\mathrm{x}$ & $\mathrm{x}$ & $\mathrm{x}$ & $x$ \\
\hline Physical examination & $x$ & & $x$ & $x$ & $x$ \\
\hline Concomitant medication & $x$ & & $x$ & $x$ & $\mathrm{x}$ \\
\hline Pregnancy test & $x$ & & & & \\
\hline Blood test & $x$ & & $\mathrm{x}$ & $\mathrm{x}$ & $\mathrm{x}$ \\
\hline ECG & $x$ & & $x$ & $x$ & $x$ \\
\hline Holter monitoring & $x$ & & & $\mathrm{x}$ & $\mathrm{x}$ \\
\hline TTE & $x$ & & & $x$ & $x$ \\
\hline Cardiac MRI with gadolinium & $\mathrm{x}$ & & & $x$ & $x$ \\
\hline HF signs and symptoms & $x$ & & $x$ & $x$ & $x$ \\
\hline Adverse events & $x$ & $\mathrm{x}$ & $x$ & $x$ & $x$ \\
\hline Surgical procedure & & $\mathrm{x}$ & & & \\
\hline
\end{tabular}

The surgical procedure will consist of a coronary artery bypass graft on the amenable area for both groups. In addition, the treatment group will undergo the adipose graft transposition procedure.

ECG, Electrocardiogram; HF, heart failure; TTE, transthoracic echocardiography

- Adverse event rate and description: arrhythmia by 24hour Holter monitoring (time frame 0-3-12 months), all cause death or readmission (time frame 12 months), and cardiovascular death or readmission (time frame 12 months).

\section{Data analyses and sample size justification}

Statistical analyses will be performed for all patients randomised and operated on. Any randomised patient who abandons the study (for any cause) before the surgical procedure will not be followed, but will be registered to investigate possible non-candidacy for the AGTP. A subgroup analysis will be performed for patients with a non-revascularisable MI $\leq 2$ months prior to surgery. The aim of this analysis is to assess the potentially increased benefit of the AGTP to this subgroup of patients in terms of remodelling and scar size/regional contractility versus patients with older chronified scars.

Descriptive analyses will be performed at the first step. Categorical variables will be described by frequencies and percentages. Continuous variables will be described by means and SD or medians and interquartile ranges in case of skewed distribution. Differences between groups will be compared using the Student's t test or one way ANOVA for multiple comparisons, with Tukey's test for post hoc analyses. Comparison of continuous variables between groups will be performed using analysis of variance for unpaired data once normality is demonstrated (Kolmogorov-Smirnov test); otherwise, a non-parametric test (Mann-Whitney or Kruskal-Wallis test) will be used. The MRI data will be analysed as repeated measures using ANOVA with Greenhouse-Geisser correction. A two sided $p<0.05$ will be considered significant. Statistical analyses will be performed using SPSS statistical package V.19.0.1 (SPSS Inc, Chicago, Illinois, USA). Monitoring will be performed by the sponsor centre, IGTP.

Accepting an alpha risk of 0.05 and a beta risk of 0.2 in a two sided test, 54 subjects are necessary in the standard of care group and 54 in the AGTP group to recognise as statistically significant a difference of $\geq 10 \%$ reduction in baseline to 12 month LGE MRI ratio change. Baseline LGE MRI ratio is estimated as $20 \% \pm 7$, according to the AGTP I trial. ${ }^{7}$ The correlation coefficient between the initial and final measurement was assumed as 0.875 . A dropout rate of $10 \%$ is anticipated.

\section{DISCUSSION}

The sequelae of MI have been decreasing since the establishment of early revascularisation procedures worldwide. However, a large number of patients still suffer from myocardial scarring, including those treated outside of the optimal reperfusion time window, or those not having viable vessels available for CABG. Currently, experimental cardiac tissue engineering approaches fall into two main categories: scaffolds and cells. Scaffolds are sometimes supplemented with cytokines, growth factors and peptides. They may be of natural or synthetic origin and 
should recreate the myocardial environment, support vascularisation, ensure electrical and mechanical properties for optimal host tissue coupling, and ultimately be degradable. Cells from several sources have been tested (ie, embryonic stem cells, adipose tissue derived stem cells, cardiac progenitor cells, skeletal myoblasts, induced pluripotent stem cells) although, to date, the optimal cell population is not identified. From a clinical perspective, the main limiting factors are the requirement for previously banked cells of autologous or allogeneic origin and, more importantly, the need for a proximal in vitro set-up outside of the confines of the operating theatre.

The AGTP is the first tissue engineering technique to use local adipose tissue without laboratory manipulation. This approach minimises the complications that can arise from the introduction of exogenous stem cells or stem cell derived cardiomyocytes, as well as those associated with gene therapy (ie, risks of contamination due to laboratory manipulation, infection, immunoreactivity, viral recombination, etc). Access to local adipose tissue is a critical and advantageous aspect of the AGTP. Adipose tissue is a natural niche of progenitor cells, with proven efficacy in tissue regeneration and disease treatment. ${ }^{9}$ Based on previous studies, the AGTP may stimulate neovascularisation and improve local microcirculation and metabolism. ${ }^{4-6}$ The paracrine effects derived from cATMSCs might also contribute to improve the microenvironment at the scar site, thereby enhancing myocardial contractility. Further enrichment with exosomes, growth factors and/or progenitor cells could form the basis of a future means of potentiating the regenerative capacity. Thus, with this trial, we could establish a new concept in cardiac tissue engineering.

To summarise, the AGTP is technically simple, requiring no learning curve for cardiac surgeons who will simply dissect structures that are ordinarily modified in CABG surgery. Furthermore, there is no requirement for additional or expensive materials, nor do any ethical or social constraints exist as the autologous tissue is sourced from a site in close proximity to the injury. ${ }^{7}$

\section{Author affiliations}

${ }^{1}$ ICREC Research Program, Fundació Institut d'Investigació en Ciències de la Salut Germans Trias i Pujol (IGTP), Badalona, Spain

${ }^{2}$ CIBERCV, Instituto de Salud Carlos III, Madrid, Spain

${ }^{3}$ Hospital Universitari Germans Trias i Pujol, Badalona, Spain

${ }^{4}$ Hospital Universitari de Girona Josep Trueta, Girona, Spain

${ }^{5}$ Hospital Universitario Puerta del Hierro, Madrid, Spain

${ }^{6}$ Hospital Universitario Central de Asturias, Oviedo, Spain

${ }^{7}$ Hospital Clínico Universitario, Valladolid, ICICORELAB, Valladolid, Spain

${ }^{8} \mathrm{Hospital}$ Clínico Universitario Virgen de la Arrixaca, Universidad de Murcia, IMIB-

Arrixaca, Murcia, Spain

${ }^{9}$ Hospital Clínico Universitario Virgen de la Victoria de Málaga, IDIMA, Málaga, Spain

${ }^{10}$ Complejo Hospitalario Universitario A Coruña (CHUAC), A Coruña, Spain

${ }^{11}$ Hospital Universitari de Bellvitge, L'Hospitalet de Llobregat, Spain

${ }^{12}$ Hospital Clínic de Valencia, Valencia, Spain

${ }^{13}$ Hospital General Universitario Gregorio Marañón, Instituto de Investigación

Sanitaria Gregorio Marañón, Madrid, Spain
${ }^{14}$ Department of Medicine, Universitat Autònoma de Barcelona, Barcelona, Spain

Contributors All authors have read and approved submission of this manuscript. All have contributed in different ways to the final version of the manuscript.

Funding The AGTP II trial is an investigator initiated study, sponsored by the Germans Trias i Pujol Research Institute. It is supported by grants from Ministerio de Educación y Ciencia (SAF2014-59892-R), Ministerio de Economía y Competitividad (Juan de la Cierva, JCI-2012-14025), Fundació La MARATÓ de TV3 $(201502,201516)$ and Generalitat de Catalunya (SGR 2014 , CERCA Programme and PERIS Programme, Departament de Salut SLT002/16/00234, SLT002_16_00209). Additional funds will be provided by Red de Terapia CelularTerCel (RD16/0011/0006) and CIBER Cardiovascular (CB16/11/00403), as part of the Plan Nacional de I+D+l, and cofunded by ISCIII-Sudirección General de Evaluación and Fondo Europeo de Desarrollo Regional (FEDER), and "La Caixa" Banking Foundation. This work is developed within the AdvanceCat project with the support of ACCIÓ (Catalonia Trade \& Investment; Generalitat de Catalunya) under the Catalonian European Regional Development Fund (ERDF) operational programme 2014-2020.

Competing interests None declared.

Patient consent Obtained.

Ethics approval The study was approved by the Germans Trias i Pujol Hospital Ethics Committee.

Provenance and peer review Not commissioned; externally peer reviewed.

Data sharing statement The article is a rationale and design manuscript. Patients are being enrolled at the moment. No further data is available yet. In addition to the paper and the protocol, the informed consent documents are available from us and from the Germans Trias i Pujol Hospital Ethics Committee. PG, JL, CGM and ABG will have access to the final trial dataset.

Open Access This is an Open Access article distributed in accordance with the Creative Commons Attribution Non Commercial (CC BY-NC 4.0) license, which permits others to distribute, remix, adapt, build upon this work non-commercially, and license their derivative works on different terms, provided the original work is properly cited and the use is non-commercial. See: http://creativecommons.org/ licenses/by-nc/4.0/

(C) Article author(s) (or their employer(s) unless otherwise stated in the text of the article) 2017. All rights reserved. No commercial use is permitted unless otherwise expressly granted.

\section{REFERENCES}

1. Olivetti G, Capasso JM, Meggs LG, et al. Cellular basis of chronic ventricular remodeling after myocardial infarction in rats. Circ Res 1991;68:856-69.

2. lacobellis G, Ribaudo MC, Assael F, et al. Echocardiographic epicardial adipose tissue is related to anthropometric and clinical parameters of metabolic syndrome: a new Indicator of cardiovascular risk. J Clin Endocrinol Metab 2003;88:5163-8.

3. Mazurek T, Zhang L, Zalewski A, et al. Human epicardial adipose tissue is a source of inflammatory mediators. Circulation 2003;108:2460-6.

4. Bayes-Genis A, Soler-Botija C, Farré J, et al. Human progenitor cells derived from cardiac adipose tissue ameliorate myocardial infarction in rodents. J Mol Cell Cardiol 2010;49:771-80.

5. Gálvez-Montón C, Prat-Vidal C, Roura S, et al. Transposition of a pericardial-derived vascular adipose flap for myocardial salvage after infarct. Cardiovasc Res 2011;91:659-67.

6. Gálvez-Montón C, Prat-Vidal C, Roura S, et al. Post-infarction scar coverage using a pericardial-derived vascular adipose flap. Preclinical results. Int J Cardiol 2013;166:469-74.

7. Bayes-Genis A, Gastelurrutia P, Cámara ML, et al. First-in-man safety and efficacy of the adipose graft transposition procedure (AGTP) in patients with a myocardial scar. EBioMedicine 2016;7:248-54.

8. Thygesen K, Alpert JS, Jaffe AS, et al. . Third universal definition of myocardial infarction. J Am Coll Cardiol 2012;60:1581-98.

9. Ma H, Liu J, Qian L. Fat for fostering: regenerating injured heart using local adipose tissue. EBioMedicine 2016;7:25-6. 